\title{
A positive attitude in prostate cancer challenges: finding hope and optimism
}

\author{
Laurence Lepherd, $\mathrm{PhD}, \mathrm{MEd},{ }^{\mathrm{ab}}$ and Coralie Graham, $\mathrm{PhD}, \mathrm{RN}^{\mathrm{c}}$
}

${ }^{\mathrm{a}}$ Centre for Health Sciences Research, University of Southern Queensland, Toowoomba, Australia; ${ }^{\mathrm{b}}$ School of Medicine, Rural Clinical School, University of Queensland, Toowoomba, Australia; 'School of Nursing and Midwifery, Faculty of Health, Engineering \& Sciences, University of Southern Queensland, Toowoomba, Australia

Background Prostate cancer affects not only men with the disease, but their partners and families as well. These affects can include changes to everyday lifestyle activities, incontinence, and sexual dysfunction, and sometimes, relationships.

Objective To find out how men with prostate cancer and their female partners found spiritual lift and hope during the prostate cancer trajectory.

Methods The very personal and human nature of the question suggested that a qualitative approach with narrative inquiry would be the most appropriate. Comments were obtained from 10 men and 10 women who were not in a relationship with each other and from 10 couples $(N=40)$ and then subjected to narrative and thematic analysis.

Results The participants' activities and circumstances provided their lift - rising above the everyday mundane - and their hope - optimism for the future - and helped them cope. In addition, what emerged was interesting insights on the way in which the participants associated these concepts with having a positive attitude in their life. They provided some valuable information on what constitutes being positive that will be helpful to others in similar circumstances, and to health professionals.

Limitations The information from a relatively small number of participants needs to be interpreted carefully and cannot result in strong conclusions about the nature of the results.

Conclusions Being positive during a time of illness and when dealing with the consequences of the illness, is an important element in coping. However, an understanding of the practicalities of what it means to be positive needs to be thoroughly developed and understood.

$\mathrm{P}$ rostate cancer has become a common disease in recent years. This could be because of increased emphasis on public awareness of screening and increased life expectancy. In Australia, for example, it is the most common cancer diagnosed. ${ }^{1}$ It is estimated that in the state of Queensland, and in Australia as a whole, 1 in 5 men will be diagnosed with prostate cancer before the age of 85 years. In Queensland, the life-time risk of a man dying from prostate cancer is 1 in 22. Prostate cancer is the second most common cause of death from cancer in men in Queensland and makes up $13 \%$ of all cancer deaths in men. The average length of survival after diagnosis with prostate cancer is 10 years. ${ }^{2,3}$ The occurrence of prostate cancer in society suggests it is important that men and those close to them develop elements of their lives that will help them lead lives that are as physically and psychologically comfortable as possible. Although the disease trajectory for prostate cancer can be very difficult for men with the disease, it can be equally difficult for their partners. Lifestyle changes such as the ability to maintain joint physically oriented activities, changes in the man's sexual ability and, sometimes, the man's emotional stability can have an impact on a couple's relationship.

Two elements of the lives of men and their partners that can assist in realising greater comfort for them are lift - the way they are able to transcend the day-to-day difficulties of the prostate cancer disease trajectory - and hope, the quality that provides expectation of comfort for them. Lift and transcendence are concepts concomitant with the notion of spirituality, the core of a person's being. ${ }^{4,5}$ Spirituality can have a positive effect on quality of life in cancer patients. ${ }^{6}$ O'Baugh and colleagues found that being positive was central to being able to cope with cancer and its treatment. ${ }^{7}$ It seems that the relationship between lift and hope, and being positive, can assist men and their partners in coping with their cancerrelated life. Spirituality (and religion) can also be associated with a positive attitude to life. ${ }^{8}$

The purpose of this study was to record the experiences of lift (spiritual) and hope in men with prostate cancer and their partners during their journey through the disease trajectory and to analyze

Accepted for publication November 16, 2015. Correspondence: Laurence Lepherd, PhD, MEd; laurence.lepherd@usq. edu.au. Disclosures: The authors report no disclosures or conflicts of interest. JCSO 2016;14(9):386-391. (C2016 Frontline Medical Communications. doi: 10.12788/jcso.0208. 
their reflections of their journey so that we would be better informed in assisting them and others in achieving or maintaining their well-being during the journey, and to provide background information for other health professionals. The research question was: How do men with prostate cancer and their partners demonstrate lift and hope during the prostate cancer journey?

We decided at the beginning of the project to focus on men with female partners rather than those with male partners to eliminate possible confounds due to partner gender in such a small study, and to limit the scope of the study to avoid having another major dimension that would add to the study's size.

\section{Method}

This research arose out of an earlier study that recognised lift and hope as dimensions of spirituality and implied that a positive approach to coping with prostate cancer could assist men and their partners in their lifestyle. ${ }^{9}$ That earlier study supported the notion that a man's partner (if he had one) - in this case, all of the partners were women - was an important element during the course of his illness, so we conducted the current study to explore the way in which lift and hope contributed to the well-being of both the man and the woman. In the current study, the premise was that lift and hope could assist in improving lifestyle, and the objective was to identify what specifically helped men and women during the disease trajectory.

Because of the deeply personal nature of the subject, we used a qualitative and narrative inquiry to investigate this. The inner experiences and feelings of each of the participants were being explored and it was seen as an advantage for participants to use their own words to express themselves. Narrative inquiry incorporates narrative analysis the construction and validation of each participant's story, including member checking ${ }^{10}$ - and thematic analysis, the identification of clusters of concepts. ${ }^{11}$ This provided the opportunity for participants to express themselves directly, and, as it happened, sometimes in intimate detail.

\section{Participants}

Three groups of participants were recruited for the study - 10 men and 10 women who were not in a relationship with each other, and 10 couples $(\mathrm{N}=40$; Table 1$)$. The men and women volunteers individually told their personal perspectives on their prostate cancer journey, often referring to their partners (who were not interviewed in the research). The couple interviews allowed for a different dynamic because the participants often interacted with each other while the story was being told. It was anticipated that interviewing couples together would provide rich data that would supplement individual interviews. ${ }^{12}$

Financial constraints determined that all the interviews were carried out by telephone rather than face-to-face. This

\begin{tabular}{lll} 
TABLE 1 Age range of participants $(\mathrm{N}=40)$ \\
Age range, $\mathbf{y}$ & Women, $\mathbf{n}$ & Men, $\mathbf{n}$ \\
$41-50$ & 1 & 1 \\
$51-60$ & 1 & 1 \\
$61-70$ & 7 & 9 \\
$71-80$ & 10 & 8 \\
\hline $81+$ & 1 & 1 \\
\hline
\end{tabular}

was productive for two reasons. It allowed the researchers to recruit participants from a broader geographical area within Australia - both rural and urban - and it allowed for an ease of contact for the participants. The narration of their stories was quite fluent for each of the participants.

The question asked at the beginning of the interview was: What gives you lift and hope during your prostate cancer journey? The interviews lasted between 14 and 55 minutes, with the average length being just under 30 minutes.

The participants were recruited by a general media release from the researchers' university, resulting in local, regional, and national newspaper articles and radio interviews, and through direct promotion through the Prostate Cancer Foundation of Australia. The criteria for the study participants were that the man or man in the relationship had experienced prostate cancer and that they were happy to share their experiences. The participants were spread geographically across Australia from Queensland and through New South Wales, Victoria, and Western Australia.

Purposive sampling was not sought to achieve representative numbers of rural or city participants or any other demographic factors. Nine of the participants lived in four state capital cities, 16 lived in rural regional cities, and five lived in rural towns. In response to the opening question, on only five occasions did participants mention location (rural or urban) as a factor in their ability to cope. The impact of time and distance from the treatment center on the participants' recollections had no effect on their stories. Some of the patients had just finished treatment, whereas others had been recovering for some time. It is important to note that the participants volunteered to tell their stories no matter how long their journey was or how long ago it was. Each person reflected as they wished on the broad issue of lift and hope and recounted their experiences. The demographic circumstances of each of the participants were notably disparate. No pattern in the results emerged from analysing the circumstances of the cohort. They were individuals whose lives were affected by the illness. They wanted to tell their story so that others might benefit.

\section{Recorded narration}

Each story was digitally audiorecorded and then transcribed, with the identity and location of the participant or 
participants changed to ensure privacy. The transcripts were then returned to the participants for member checking to ensure the accuracy of transcription, and that on reflection they were satisfied with their stories. The narrative analysis of the interviews was designed to allow the participants to confirm the construction of their stories.

After the transcripts had been returned, the researchers undertook thematic analysis. This occurred in 2 steps. First, all of the transcriptions were imported into NVivo software (QSR International, Doncaster, Victoria, Australia) and a word frequency analysis was done. Words that were specifically relevant and used frequently were documented as Nodes. Frequently used words and their contexts were inserted into a data (text) document that acted as a "receptacle" for the participants' thematic statements. Second, each story was re-read and manually checked for themes and related matter. Anything deemed relevant that had not been included in the NVivo analysis was added to the main data document.

\section{Results}

\section{The term, positive}

While spiritual lift and hope were important themes for the participants, it was noted that the term positive was frequently used in association with the other two terms, and often used independently. Following are the results of the analysis that relate to being positive. (The occasional occurrence of "positive" in the context of "the test came back positive" has been omitted from references below). The positive subtheme usually had a number of descriptors but reporting the results starts with the word used without qualification - where being positive had a simple implication of being optimistic. The next descriptor covers the issue of men and women being positive and stating their reasons for this. Then follows a description of their positivity because of the support they received. Statements of the way they felt helped by their positivity are then included, and lastly, statements on the way some of the participants believed they experienced growth through being positive are recorded. (Recorded statements were made by men unless indicated by $w$, for women.)

Positive - optimism implied. The men and their partners frequently spoke of being positive in a general optimistic sense. For example, "Alan was so positive about the whole thing" and "I've always been a very positive person." The phrase, "I'm a very positive person" was used by four different people.

For one couple, being positive resulted from an improvement in circumstances implying that dark circumstances can lead to a lack of optimism. The woman in the couple said,

I am beginning to feel that there is a shift, and that things are now more positive - blue sky ... I just wish for him that there can be something positive. We actually had to start to spend time exclusively with one another and push other people away and focus on ourselves, which is at odds with both of us because we are both very giving and very generous people.

Positive - reason for optimism. A number of participants provided reasons for their optimism. These included a man being able to drive himself to and from his treatment (radiation), "getting through things," and a man going to the gym "for the first time in his life," having counselling, and confidence that his ultimate conclusion "should be fine," especially if he had a positive attitude.

Other reasons included, work experiences that prepared one man for his cancer experience. "Keeping busy" was a factor in another man's life. One man's partner's support through her understanding, as a nurse, of hospital procedures was "a big positive reality." Another dimension was that a partner made herself keep up "a positive attitude for him and the children." Others attributed their positive approach to their Christian faith or spiritual belief.

There was an inverse positivity about one partner's response: "We are very positive about it all now because we haven't had anything horrific happen." Another recognised that observing older couples get through the illness had been a positive inspiration for him,

So this was a case of older people getting through it and showing that getting through it is a possibility for younger people. This gave me great positive hope.

A more substantial reason for being positive was expressed by one woman in these terms,

I'm lucky that my husband is such a positive person and he is my rock, and I suppose I am his rock as well. ... We have decided that life is too short to dwell on what could have been, and now we just live each day as best we can.

Some had reasons for their positive approach because they had received good test results and felt "quite positive at this stage." Implied was that they might not have felt so positive if the results had been less favourable. Another partner commented,

There can be arguments as to why he is feeling despondent, and why he is not feeling positive when his diagnosis is not good.

In speaking of some of the health difficulties experienced in her family, one woman commented,

I think this is another indication that we look at positive 
things. Things you don't want sometimes happen but we confront them when they come.

Positive - support. Most of the participants had experienced considerable support from many sources that helped them to be positive. These included, "We have very positive friends who have gone down the same track," and "Everybody else was straight down the line and very positive, including the urologist," and "Right from the get-go the urologist and the GP, and the oncologist, were all very positive."

Some participants stressed the importance of being with positive people. One said the reason "I still go to my cancer support group is to be around positive people." Positive support people included doctors, family members, mixed support groups, and partner support groups, although sometimes a man or his partner did not want to be part of a support group. One woman said,

I've never had the need for a partner support group because I'm a very positive and determined sort of person and also very private.

Positivity - helped by. Some participants were helped by a positive approach, which they hinted through comments such as, "I think my positive attitude has helped me absolutely" and "We were all positive, which helped me quite a lot." Some women participants were also helped by their partners' positive approach, "He seemed to be quite positive and taking it just one day at a time. This was very helpful to me," as were some couples, "We definitely have a positive attitude and this has helped us as a couple."

Other instances of help include physical and mental activity,

I have found that exercise and meditation, and being around positive people, has helped me cope immensely.

In another example, one man indicated that he was helped by being positive, but not without a struggle,

I think I was wafting in and out of it a bit and not being positive with myself about it ... it has taken a while, but I am much more able to make positive decisions in an easy manner. I was reasonably positive from the point of view that I'm the sort of individual whose glass is half full rather than being half empty. That probably helped me cope with that too.

Positive - personal growth. One of the most telling outcomes of being positive was that a number of participants had personal growth through this. One woman said, "It has caused us to find the very best we can out of adverse circumstances. That's the positive." Others said, "We are both pretty positive people most of the time and we just got on with it" and "I'm very positive about the future for him and for us, and we are going forward with it all."

For some participants, being realistic was a vital part of their growth. They recognised that, for them, it was important to be positive, but this had to be colored by realism,

This independence has helped me to always look on the positive side of things. I have never had the issue of, "Oh, oh, oh, what could go wrong" type of thing. My positive attitude gives me a lift; I'm a realistic person.

One man saw his cancer as an important part of his own life journey but not the only part. He believed he had coped with the cancer component of his life but there were other things in his life in which he need personal growth, "My long-term health looks positive, it's the rest of my life I need to work on." There was little doubt that there was a benefit in a mutual positive approach to the issue, at least for one woman,

I was always on the positive side because he was so positive. This helped me to get through what we were both going through. It has bolstered my approach to the difficulty.

Being positive and having hope. One woman summed up the feelings of a number people,

\begin{abstract}
We have a very positive hope for the future. We have great hope; we just get on with things. We are Christians and we know everything will be fine, whatever it is, and we will get through it. We are not seeing 'pie-in-the-sky'. You face the reality of what may, or what may not happen, but is not much point spending the rest of your life dwelling on that; we just think positive. We have a sound relationship and good family and good friends and we don't need absolutely anything more than that."
\end{abstract}

A summary of some of some participants' key statements about being positive and their reasons for being positive is shown in Table 2.

\section{Discussion}

In this article, we explored the positive aspects of men's and women's experiences during the prostate cancer trajectory. Of particular note is the value of being positive during the journey, as reflected through the spontaneous use of the word in the participants' stories, and that on the whole, they indicated what being positive meant for them, often in very direct terms.

Having a positive attitude is often regarded as being important in health treatment and is commonly used as a way of coping as reported by Wilson ${ }^{13}$ and $\mathrm{O}^{\prime} \mathrm{Baugh}^{14}$ and 
TABLE 2 Statements about being positive, with the reasons (in italic)

We are very positive about it all now because we haven't had anything horrific happen.

Another thing about positive support aspects was that about 12 months ago I started going along to the gym for the first ever time in my life.

I also admit that I tried to keep myself busy and keep that positive attitude towards everything.

... everyone was taking a positive approach that the treatment would be successful.

The positive attitude that I have, and my lift, is basically through my beliefs and my faith.

The journey has probably strengthened our relationship and this is the great positive that has come out of it.

This to me is a big positive reality. Understanding hospital procedures etc is second nature to her and she was able to translate that to me.

One of his brothers is a complete tissue-type if ever he needs a bone marrow transplant, so it is all positive.

We have a very positive hope for the future. We have great hope; we just get on with things.

their colleagues, although they also indicate that it is a very difficult concept to define (O'Baugh, p. 113). Wilson and colleagues do, however, cite some comments by patients and nurses in their study,

Patients defined 'being positive' as maintaining some sort of normality without letting cancer have a detrimental effect on daily living. Nurses identified hope, acceptance, fighting spirit and looking on the bright side as definitions of 'being positive.' (p. 62)

However, Coyne and colleagues ${ }^{15}$ questioned the notion that being positive and having a "fighting spirit" helps in physical recovery and during post-traumatic growth. They found that there was very dubious evidence of a relationship between being positive and improvement in health, a relationship sometimes promoted in research and literature. They concluded that there needed to be more scientific evidence promoting the value of positive psychology. Similar reservations about the value of hope in illness have been expressed by Garret. ${ }^{16}$ Those findings notwithstanding, the perceptions of the participants in our current study were very much in favor of linking a positive attitude to a greater feeling of comfort during their journey through the disease trajectory. It is significant that none of our participants expressed the view that being positive would prolong life or improve physical health.
Of particular significance is what the participants regarded, as indicated in the stories they told, as contributing to their positive attitude. Defining this concept - that is, what helps to build a positive attitude in a way that might also be helpful to others? - is not always evident in existing literature aimed at helping others face these challenges. For example, one organisation suggests that men with prostate cancer should "have a strong positive attitude and plan for the future," ${ }^{17}$ but there is no indication of how that might be achieved. This absence of a description of what assists in the development of a positive attitude seems to be common in a number of published papers. ${ }^{18-20}$ While it may be important to encourage men and woman in difficult circumstances to adopt a positive attitude to assist in their coping, it would be helpful to indicate to them the practical and spiritual activities of their lives that might help them achieve that.

Participants in the current study were found to embrace the notion of being positive in their approach to prostate cancer and related that notion to the concepts of lift and hope. They frequently had reasons for being positive that extrinsically included interpersonal relationships and support from a range of people and intrinsically included faith and belief as well as hope and altruistic pursuits of helping others for the betterment of other men and women whose lives would be challenged by prostate cancer.

\section{Limitations}

The information from this relatively small number of participants needs to be interpreted carefully and cannot result in strong conclusions about the nature of the results. ${ }^{21}$ That the participants volunteered to tell their stories relating to lift and hope may well have skewed the sample and excluded those who did not experience lift and hope during their journey. This research concludes that for the men and women interviewed, their stories provide their personal perspectives. Although these perspectives may be similar to others, they are not necessarily so. That the participants volunteered their stories suggests that they had something they wanted to say and this may differ from a more general population of partners who did not volunteer to be interviewed or express themselves at all. Ultimately, that these participants, as individuals, had these experiences indicates that it is possible for the experiences to exist in others.

\section{Conclusions}

One essential element of the findings of this research is that lift, hope and positivity are often achievable aspects of a life related to prostate cancer but must be tempered by reality. Some participants demonstrated that it was not always possible to achieve lift and hope and to be positive. This was usually determined by the extent of the severity of their illness and other life circumstances. They perceived, 
however, that being positive could help them during their journey.

This information might be used by other people in similar circumstances, and health professionals in a variety of disciplines, to understand more fully the way people can experience greater comfort during their journey. An important consideration is that practical examples of what it means to be positive should be provided to those with, and associated with the illness to indicate that a positive atti-

\section{References}

1. Prostate Cancer Foundation of Australia. 08/09 collaborative research update. http://www.prostate.org.au/publications/collaborativeresearch-updates/. Published 2008. Accessed September 12, 2016

2. Australian Institute of Health and Welfare \& Australasian Association of Cancer Registries. Cancer in Australia: an overview 2008. Canberra: Australian Institute of Health and Welfare; 2008.

3. Queensland Cancer Registry. Cancer in Queensland: incidence and mortality 1982 to 2006. Statistical Tables. Spring Hill: Queensland Cancer Registry and Cancer Council Queensland; 2009.

4. Miner-Williams D. Connectedness in the nurse-patient relationship: a grounded theory study. Issues Ment Health Nurs. 2007;28:1215-1234.

5. Schultz N. The spiritual dimension in nursing care. In: Rogers-Clark C, McCarthy A, Martin-McDonald K, eds. Living with illness: psychosocial challenges for nursng. Marrickville, NSW, Australia: Elsevier; 2005. pp 160-170.

6. Daugherty CK, Fitchett G, Murphy PE, et al. Trusting God and medicine: spirituality in advanced cancer patients volunteering for clinical trials of experimental agents. Psychooncology. 2005;14(2):135-146.

7. O’Baugh J, Wilkes LM, Luke S, George A. 'Being positive': perceptions of patients with cancer and their nurses. J Adv Nurs. 2003;44(3):262-270.

8. Lukoff D. DSM-IV religious \& spiritual problems [no date; cited September 4, 2009]. http://www.spiritualcompetency.com/dsm4/ dsmrsproblem.pdf. Accessed September 12, 2016.

9. Lepherd L. Spirituality in men with advanced prostate cancer: 'It's a holistic thing ... it's a package'. J Holist Nurs. 2014;32(2):89-101.

10. Angen MJ. Evaluating interpretive inquiry: reviewing the validity debate and opening the dialogue. Qual Health Res. 2000;10(3):378-395. tude might be achieved through being optimistic, having a reason for optimism, helping and being helped, spiritual belief and having support facilities.

\section{Acknowledgments}

The administrative support and promotional assistance of the Prostate Cancer Foundation of Australia is gratefully acknowledged. This research was approved by the University of Southern Queensland Human Research Ethics Committee.

11. Madill A, Gough B. Qualitative research and its place in psychological science. Psychol Methods. 2008;13(3):254-271.

12. Bjørnholt M, Farstad GR. 'Am I rambling?' on the advantages of interviewing couples together. Qual Res. 2014;14(1):3-19.

13. Wilson SE, Andersen MR, Meischke H. Meeting the needs of rural breast cancer survivors: what still needs to be done? J Womens Health Gend Based Med. 2000;9(6):667-677.

14. O'Baugh J, Wilkes LM, Luke S, George A. Positive attitude in cancer: the nurse's perspective. Int J Nurs Pract. 2008;14:109-114.

15. Coyne JC, Tennen H. Positive psychology in cancer care: bad science, exaggerated claims, and unproven medicine. Ann Beh Med. 2010;39:16-26.

16. Garrett C. Sources of hope in chronic illness. TASA Conference; University of Sydney 2001

17. National Seniors Foundation. Localised prostate cancer - a guide for men and their families. Spring Hill, Brisbane: Australian Prostate Cancer Collaboration Inc; 2006.

18. Gall TL, Kristjansson E, Charbonneau C, Florack P. A longitudinal study on the role of spirituality in response to the diagnosis and treatment of breast cancer. J Behav Med. 2009;32:174-186.

19. Gall TL, Cornblat MW. Breast cancer survivors give voice: a qualitative analysis of spiritual factors in long-term adjustment. Psychooncology. 2002;11(6):524-535.

20. Lev EL, Sanzero Eller L, Gejerman G, et al. Quality of life of men treated with brachytherapies for prostate cancer. http://hqlo.biomedcentral.com/articles/10.1186/1477-7525-2-28. Published June 15, 2004. Accessed September 13, 2016.

21. Hackshaw A. Small studies: strengths and limitations. Eur Respir J. 2008;32:1141-1143. 